\title{
Presencia de los géneros invasores Mus y Rattus en áreas naturales de Chile: un riesgo ambiental y epidemiológico
}

\author{
Presence of the invasive genera Mus and Rattus in natural areas in Chile: \\ an environmental and epidemiological risk
}

GABRIEL LOBOS ${ }^{1}$, MARCELA FERRES $^{2} \&$ R. EDUARDO PALMA ${ }^{13}$

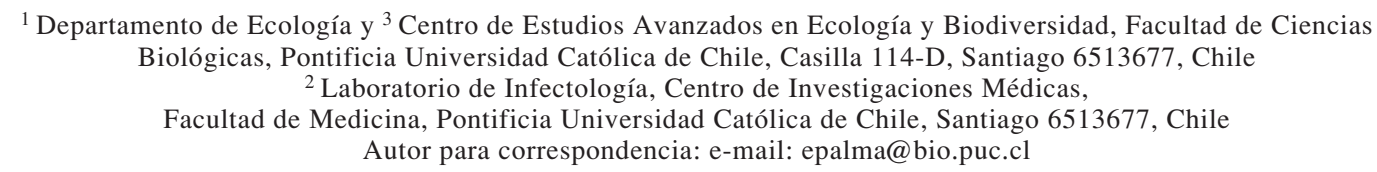

\section{RESUMEN}

Realizamos un estudio que incluyó muestreos y prospecciones en un gradiente latitudinal en Chile continental para determinar la presencia y ausencia de roedores murinos introducidos, particularmente Mus musculus, Rattus rattus y $R$. norvegicus en áreas naturales o silvestres a lo largo de Chile. Además se analizó el riesgo epidemiológico que representan estas especies en el marco de un estudio sobre el virus Hanta. Los resultados mostraron que M. musculus rara vez es recolectado en áreas naturales. Sin embargo, las dos especies de Rattus han invadido ampliamente la región mediterránea chilena. Las regiones desérticas, los ambientes de alturas y las regiones australes, serían biótopos restringidos para estos invasores. Desde una perspectiva epidemiológica, la presencia del virus Hanta (variedades Andes y Seoul) en Rattus es un elemento que demuestra que las especies invasoras además de generar impactos ecológicos, pueden ocasionar problemas económicos y de salud pública. La fragilidad de los ecosistemas mediterráneos determina que la presencia de especies exóticas constituya un elemento de alto riesgo para la conservación del patrimonio natural del país. Probablemente, la conservación de áreas naturales constituye la mejor herramienta para enfrentar a estas especies exóticas.

Palabras clave: Mus musculus, Rattus rattus, Rattus norvegicus, especies invasoras, virus Hanta Andes, Virus Hanta Seoul, Chile.

\begin{abstract}
We conducted a latitudinal study in natural areas of continental Chile to evaluate the occurrence of the introduced murine rodents Mus musculus, Rattus rattus and R. norvegicus. Furthermore, we evaluated the epidemiological risk of these species as part of an ongoing study on Hantavirus. The results allowed us to conclude that $M$. musculus occurs rarely in natural environments. However, the two species of Rattus have widely invaded the mediterranean region of Chile. Desert, altitudinal and high latitude regions seem to be restricted areas for these invasive rodents. From an epidemiological perspective, the occurrence of Hantavirus in Rattus (Andes and Seoul strains) other than cause an ecological impact to the ecosystems, can create economic and public health disturbances. The fragility of mediterranean ecosystems shows that the presence of exotic species constitutes an element of risk for the conservation of the natural areas in Chile. Probably, the conservation of natural environments will constitute the best tool to manage these exotic species.
\end{abstract}

Key words: Mus musculus, Rattus rattus, Rattus norvegicus, invader species, Andes Hanta virus, Seoul Hanta virus, Chile.

\section{INTRODUCCIÓN}

Las invasiones biológicas representan una seria amenaza para la biodiversidad (Rodríguez 2001). Los efectos, directos e indirectos, que las especies invasoras pueden ocasionar al me- dio ambiente, han sido tema importante en la biología de la conservación (Sutherland 2001). Para Chile se reportan 24 especies de vertebrados invasores (Jaksic 1998), desconociéndose en muchas de ellas el impacto que estas tienen en los ecosistemas invadidos. La depredación, 
la transmisión de parásitos y enfermedades, la modificación del hábitat, así como la alteración de redes tróficas son algunos de los impactos que pueden ser atribuidos a las especies invasoras (Crossland 2000, Kats \& Ferrer 2003, Mazzoni et al. 2003, Camus 2005).

Los roedores murinos cosmopolitas (Mus musculus, Rattus rattus y $R$. norvegicus) tienen como distribución original el área Indo-Asiática (Núñez \& Cisternas 1991, Aplin et al. 2002). Mus Musculus y R. rattus fueron transportados a Europa durante las cruzadas (siglo XIII), mientras que $R$. norvegicus se dispersó a este continente de manera natural en el mismo siglo, diezmando las poblaciones de $R$. rattus (Campos 1996). Para Chile, estos roedores son las especies invasoras más antiguas. Rattus rattus y Mus musculus habrían llegado con los conquistadores españoles en el siglo XVII. La invasión posterior de $R$. norvegicus a Europa determinó que esta especie llegara a las Américas en el siglo XIX (Jaksic 1998). Desde entonces en Chile, pocos estudios se han realizado en aspectos ecológicos de estos roedores. La mayoría de las investigaciones se han centrado en los daños económicos (e.g., pérdida de cosechas) y epidemiológicos que estos roedores causan en los ambientes antrópicos (Núñez \& Cisternas 1991, Rodríguez 1993).

Para Chile se señala que los roedores murinos están presentes en todo el país, asociados preferentemente a poblaciones humanas (Miller \& Rottmann 1976, Pine et al. 1979, Campos 1996, Muñoz-Pedreros \& Yáñez 2000). Muy pocos estudios han documentado la situación de poblaciones silvestres (Pefaur et al. 1978, Simonetti 1983) y en algunos casos se citan datos anecdóticos de presencia en ambientes naturales (Figueroa et al. 2001). Existe escasa información sobre la distribución de estas especies en ambientes naturales y el impacto que pudieran ocasionar en ellos. El conocimiento de que normalmente las dos especies de Rattus atacan huevos y aves en graneros, ha sugerido que lo mismo puede suceder en ambientes naturales. No obstante, el impacto de estas especies sobre fauna autóctona ha sido ampliamente documentado en islas oceánicas (Bell 2002, García et al. 2002, Micol \& Jouventin 2002, Robertson \& Saul 2004).

En este estudio reportamos los resultados de un estudio latitudinal que permitió determinar presencia y ausencia de roedores murinos en áreas naturales o silvestres a lo largo de Chile. Además analizamos el riesgo epidemiológico que representan estas especies en el marco de un estudio sobre el virus Hanta como enfermedad emergente (Schmaljohn \& Hjelle 1997).

\section{MATERIALES Y MÉTODOS}

\section{Área de estudio}

Entre los años 2000 y 2004 se realizaron capturas periódicas de roedores en el marco de un estudio sobre la ecología y epidemiología del virus Hanta en Chile, entre las regiones I-XII (Torres-Pérez et al. 2004, Palma et al. resultados no publicados). Como parte de este último estudio se muestrearon 54 localidades a lo largo de Chile para analizar la dinámica espacial y la presencia de anticuerpos al virus Hanta en micromamíferos silvestres. En la selección de estas localidades se consideraron dos aspectos: representatividad de la mayor cantidad de ecorregiones presentes en Chile (Di Castri 1968), y naturalidad de los mismos. Este último aspecto se refiere a la ausencia de asentamientos humanos (aldeas, pueblos, ciudades) en los lugares de muestreo, y a la presencia de vegetación nativa. Dichos muestreos fueron realizados al menos una vez por mes. Adicionalmente, dentro del área de estudio se seleccionaron tres sitios de marcaje y recaptura de pequeños mamíferos que representan tres de las principales ecorregiones en Chile: San Carlos de Apoquindo (región mediterránea), Parque Nacional Villarrica (Bosques Templados), y Monumento Natural Dos Lagunas (Bosque Templado Frío). Estos sitios fueron monitoreados de manera intensiva (cada 60 días) para evaluar la dinámica poblacional de roedores y pequeños mamíferos y la seropositividad al virus Hanta. San Carlos de Apoquindo (Región Metropolitana) se ubica en los contrafuertes cordilleranos de la región mediterránea de Chile central, muy próxima a la ciudad de Santiago. Presenta una vegetación característica de la zona con bosque de formación esclerófila, con presencia de especies tales como Cryptocarya alba, $\mathrm{Li}$ threa caustica y Quillaja saponaria. El Parque Nacional Villarrica, sector Quetrupillan (IX Región), corresponde a un área representativa de bosques templados del sur de Chile situada 40 $\mathrm{km}$ al sureste de la ciudad de Pucón. La vegetación es predominantemente boscosa destacando 
especies como Drimys winteri, Nothofagus alpina y N. dombeyi. El Monumento Natural Dos Lagunas (XI Región), ubicado a unos $45 \mathrm{~km}$ de la ciudad de Coyhaique, se caracteriza por tener una vegetación representativa de bosques caducifolios destacándose las especies Nothofagus antarctica y $N$. pumilio, así como de estepas frías donde predomina Stipa sp. y Mulinum sp. Todos los sitios muestreados fueron georreferenciados con un GPS (Global Positioning System), se caracterizó su vegetación, se registró su altitud, y se estimaron las distancias a asentamientos humanos a través del uso de Sistemas de Información Geográfica (software ArcView 3.1).

\section{Capturas}

Para las capturas de roedores se utilizaron trampas Sherman $(8 \times 9 \times 23 \mathrm{~cm})$ cebadas con una mezcla de avena, atún y vainilla. El protocolo de capturas consideró dos tipos de recolectas: (1) en las tres localidades de colecta intensiva se realizaron muestreos mes por medio, lo que permitió un análisis de los datos en el tiempo. En cada una de estas localidades se establecieron tres sitios fijos en los que se instalaron 160 trampas-noche por cuatro noches (a excepción de San Carlos de Apoquindo donde se instalaron dos sitios); (2) para el resto del país, se realizaron estudios de remoción con un número variable de trampas, dependiendo de las características del lugar, generalmente por tres noches. La densidad relativa de las capturas de roedores se expresa como el número de capturas/número total de trampas noche x 100 (Torres-Pérez et al. 2004).

\section{Diagnóstico de seropositividad al virus Hanta}

A todos los murinos capturados (161 especímenes, ver Resultados) se les extrajo muestras de sangre y órganos en terreno (estas últimas muestras solo para estudios de remoción), de acuerdo al protocolo del "Center for Desease Control and Prevention" (Mills et al. 1995). Las muestras fueron transportadas en nitrógeno líquido al Laboratorio de Infectología del Centro de Investigaciones Médicas de la Pontificia Universidad Católica de Chile. La búsqueda de anticuerpos se realizó mediante la técnica del "Strip Blot Inmune Assay" (SIA) para virus Hanta variedad Andes, del sur de Sudamérica, y Virus Hanta variedad Seoul. Esta última cepa corresponde a la variante presente en la zona
Indo-Asiática, Europa y Estados Unidos, de la cual Rattus constituye el reservorio natural (Hart \& Bennett 1999).

\section{RESULTADOS}

\section{Distribución latitudinal}

En relación a Mus musculus (laucha), se recolectó un total de 24 especímenes. De las tres especies de murinos, M. musculus fue la que estuvo más fuertemente asociada al hombre. $\mathrm{Su}$ presencia en ambientes naturales se restringe a Lasana (II Región) y un episodio de apariciones en Chile central durante el año 2000 (Tabla 1). Ninguno de los registros de M. musculus en ambientes naturales aparece asociado a la presencia de Rattus. Mus musculus fue recolectado solo en cinco localidades en un gradiente altitudinal que abarca entre los 670 a $2.650 \mathrm{~m}$. De los tres murinos, M. musculus fue el que se recolectó más cerca de asentamientos humanos $(\overline{\mathrm{x}}$ $\pm \mathrm{DE}=4,6 \pm 3,9 \mathrm{~km}$, distancia mínima $=2 \mathrm{~km}$, distancia máxima $=10 \mathrm{~km})($ Tabla 2).

De la especie Rattus rattus (rata negra) se recolectaron 57 ejemplares de 10 localidades entre las regiones IV y X (Tabla 2). Rattus rattus ocupó una gran variedad de ambientes a lo largo de Chile. Las dos especies de Rattus fueron recolectadas en el mismo lugar solo cuando estaban presentes en baja densidad (Tabla 1). Por lo general, cuando atrapamos sobre tres ejemplares de una especie, la otra especie no apareció. Ambas formas fueron recolectadas entre los 10 y $1.297 \mathrm{~m}$ de altitud. Las áreas invadidas por esta especie se encuentran más alejadas de asentamientos humanos, en comparación a $\operatorname{Mus}(6,9 \pm 4,1 \mathrm{~km}$, distancia mínima $=$ $2 \mathrm{~km}$, distancia máxima $=14 \mathrm{~km})($ Tabla 2$)$.

En cuanto a Rattus norvegicus (rata noruega), se recolectaron ochenta especímenes, en 16 localidades entre las regiones III-X (Tabla 2). Al igual que su congénere, esta especie ocupó una amplia variedad de ambientes naturales. Del mismo modo, la alta densidad de uno de los Rattus estuvo asociada a una baja densidad o ausencia de la otra especie del género. Se distribuyó altitudinalmente entre los 10 y $1.300 \mathrm{~m}$. De los tres murinos estudiados, esta especie fue la que más se alejó de asentamientos humanos $(7,7 \pm 5,9 \mathrm{~km}$, distancia mínima $=1 \mathrm{~km}$, distancia máxima $=20 \mathrm{~km})($ Tabla 2$)$. 


\section{TABLA}

Localidades con la densidad relativa de roedores murinos recolectados en Chile. Latitud (LAT) y longitud (LONG) en grados, altura (ALT) en m. Mus musculus (Mmus), Rattus rattus (Rrat) y $R$. norvegicus (Rnor)

Localities with the relative density where murine rodents have been captured in Chile. Latitude (LAT) and longitude (LONG) in degrees, altitude (ALT) in m. Mus musculus (Mmus), Rattus rattus (Rrat) and R. norvegicus (Rnor)

\begin{tabular}{|c|c|c|c|c|c|c|c|c|c|}
\hline \multirow[t]{2}{*}{ Localidad } & \multirow[t]{2}{*}{ Región } & \multirow[t]{2}{*}{ LAT } & \multirow[t]{2}{*}{ LONG } & \multirow[t]{2}{*}{ ALT } & \multirow[t]{2}{*}{ Fecha } & \multirow{2}{*}{$\begin{array}{r}\text { Trampas } \\
\text { noche }\end{array}$} & \multicolumn{3}{|c|}{ Densidad relativa } \\
\hline & & & & & & & Mmus & Rrat & Rnor \\
\hline Lasana, río Loa & II & $-22,16$ & 68,37 & 2.650 & Nov-03 & 30 & 13,3 & & \\
\hline Valle del río Tránsito & III & $-28,53$ & $-70,17$ & 1.300 & Nov-02 & 10 & & & 10,0 \\
\hline Parque Nacional Fray Jorge & IV & $-30,67$ & $-71,64$ & 265 & Sep-00 & 150 & & & 11,3 \\
\hline Ñague, Los Vilos & IV & $-31,84$ & $-71,52$ & 78 & Jun-03 & 720 & & 0,3 & 0,1 \\
\hline Quebrada de Córdova & $\mathrm{V}$ & $-33,44$ & $-71,65$ & 10 & Jul-03 & 360 & & 0,3 & 0,3 \\
\hline Huechún & $\mathrm{RM}$ & $-33,02$ & $-70,82$ & 670 & Dic-00 & 514 & 1,7 & & \\
\hline San Carlos de Apoquindo Sitio 2 & $\mathrm{RM}$ & $-33,28$ & $-70,29$ & 1.297 & Ene-00 & 640 & 0,2 & & \\
\hline San Carlos de Apoquindo Sitio 1 & $\mathrm{RM}$ & $-33,24$ & $-70,29$ & 1.248 & Mar-00 & 640 & & & 0,2 \\
\hline San Carlos de Apoquindo Sitio 2 & $\mathrm{RM}$ & $-33,28$ & $-70,29$ & 1.297 & Mar-00 & 640 & 0,2 & & \\
\hline San Carlos de Apoquindo Sitio 1 & $\mathrm{RM}$ & $-33,24$ & $-70,29$ & 1.248 & May-00 & 640 & 1,3 & & \\
\hline San Carlos de Apoquindo Sitio 2 & $\mathrm{RM}$ & $-33,28$ & $-70,29$ & 1.297 & Ene-01 & 640 & & 0,8 & \\
\hline San Carlos de Apoquindo Sitio 1 & $\mathrm{RM}$ & $-33,24$ & $-70,29$ & 1.248 & Mar-01 & 640 & & 0,8 & \\
\hline San Carlos de Apoquindo Sitio 2 & $\mathrm{RM}$ & $-33,28$ & $-70,29$ & 1.297 & Mar-01 & 640 & & 0,2 & \\
\hline San Carlos de Apoquindo Sitio 1 & $\mathrm{RM}$ & $-33,24$ & $-70,29$ & 1.248 & May-01 & 640 & & & 0,2 \\
\hline San Carlos de Apoquindo Sitio 2 & $\mathrm{RM}$ & $-33,28$ & $-70,29$ & 1.297 & May-01 & 640 & & 0,2 & \\
\hline San Carlos de Apoquindo Sitio 2 & $\mathrm{RM}$ & $-33,28$ & $-70,29$ & 1.297 & Jul-01 & 640 & & & 0,2 \\
\hline San Carlos de Apoquindo Sitio 1 & $\mathrm{RM}$ & $-33,24$ & $-70,29$ & 1.248 & Sep-01 & 640 & & 0,2 & 0,2 \\
\hline San Carlos de Apoquindo Sitio 2 & $\mathrm{RM}$ & $-33,28$ & $-70,29$ & 1.297 & Sep-01 & 640 & & 0,3 & 0,2 \\
\hline San Carlos de Apoquindo Sitio 1 & $\mathrm{RM}$ & $-33,24$ & $-70,29$ & 1.248 & Ene-02 & 640 & & 0,2 & \\
\hline San Carlos de Apoquindo Sitio 2 & $\mathrm{RM}$ & $-33,28$ & $-70,29$ & 1.297 & Ene-02 & 640 & & 0,2 & 0,2 \\
\hline San Carlos de Apoquindo Sitio 1 & $\mathrm{RM}$ & $-33,24$ & $-70,29$ & 1.248 & Mar-02 & 640 & & & \\
\hline San Carlos de Apoquindo Sitio 2 & $\mathrm{RM}$ & $-33,28$ & $-70,29$ & 1.297 & Mar-02 & 640 & & 0,2 & \\
\hline San Carlos de Apoquindo Sitio 1 & $\mathrm{RM}$ & $-33,24$ & $-70,29$ & 1.248 & May-02 & 640 & & 0,2 & 0,2 \\
\hline San Carlos de Apoquindo Sitio 2 & RM & $-33,28$ & $-70,29$ & 1.297 & May-02 & 640 & & & 0,2 \\
\hline San Carlos de Apoquindo Sitio 1 & $\mathrm{RM}$ & $-33,24$ & $-70,29$ & 1.248 & Jul-02 & 640 & & 0,2 & \\
\hline San Carlos de Apoquindo Sitio 1 & $\mathrm{RM}$ & $-33,24$ & $-70,29$ & 1.248 & Sep-02 & 640 & & 0,2 & 0,2 \\
\hline San Carlos de Apoquindo Sitio 2 & $\mathrm{RM}$ & $-33,28$ & $-70,29$ & 1.297 & Sep-02 & 640 & & & 0,3 \\
\hline San Carlos de Apoquindo Sitio 1 & $\mathrm{RM}$ & $-33,24$ & $-70,29$ & 1.248 & Nov-02 & 640 & & & 0,2 \\
\hline San Carlos de Apoquindo Sitio 1 & $\mathrm{RM}$ & $-33,24$ & $-70,29$ & 1.248 & Ene-03 & 640 & & & 0,2 \\
\hline San Carlos de Apoquindo Sitio 2 & $\mathrm{RM}$ & $-33,28$ & $-70,29$ & 1.297 & Ene-03 & 640 & & & 0,3 \\
\hline San Carlos de Apoquindo Sitio 2 & $\mathrm{RM}$ & $-33,28$ & $-70,29$ & 1.297 & Mar-03 & 640 & & & 0,3 \\
\hline San Carlos de Apoquindo Sitio 2 & $\mathrm{RM}$ & $-33,28$ & $-70,29$ & 1.297 & May-03 & 640 & & & 0,2 \\
\hline San Carlos de Apoquindo Sitio 2 & $\mathrm{RM}$ & $-33,28$ & $-70,29$ & 1.297 & Ene-04 & 640 & & & 0,2 \\
\hline San Carlos de Apoquindo Sitio 1 & $\mathrm{RM}$ & $-33,24$ & $-70,29$ & 1.248 & Mar-04 & 640 & & & 0,2 \\
\hline El Canelo & $\mathrm{RM}$ & $-33,33$ & $-70,24$ & 1.190 & Ene-04 & 255 & & 0,8 & \\
\hline El Ingenio & $\mathrm{RM}$ & $-33,47$ & $-70,14$ & 1.296 & Dic-02 & 300 & & & 0,3 \\
\hline Rinconada de Maipú & $\mathrm{RM}$ & $-33,50$ & $-70,91$ & 670 & May-00 & 823 & 0.1 & & \\
\hline Rinconada de Maipú Sitio 1 & $\mathrm{RM}$ & $-33,50$ & $-70,91$ & 670 & Jul-01 & 360 & & 0,3 & 0,6 \\
\hline Rinconada de Maipú Sitio 2 & $\mathrm{RM}$ & $-33,50$ & $-70,91$ & 670 & Jul-01 & 120 & & & 0,8 \\
\hline Rinconada De Maipú Sitio 3 & $\mathrm{RM}$ & $-33,50$ & $-70,91$ & 670 & Jul-01 & 135 & & 0,7 & \\
\hline El Toyo & $\mathrm{RM}$ & $-33,60$ & $-70,38$ & 928 & Feb-01 & 180 & & & 5,6 \\
\hline Alhué Sitio 1 & $\mathrm{RM}$ & $-33,95$ & $-71,03$ & 568 & Ago-03 & 320 & & 0,3 & 1,3 \\
\hline
\end{tabular}


TABLA 1 (continuación)

\begin{tabular}{|c|c|c|c|c|c|c|c|c|c|}
\hline \multirow[t]{2}{*}{ Localidad } & \multirow[t]{2}{*}{ Región } & \multirow[t]{2}{*}{ LAT } & \multirow[t]{2}{*}{ LONG } & \multirow[t]{2}{*}{ ALT } & \multirow[t]{2}{*}{ Fecha } & \multirow{2}{*}{$\begin{array}{r}\text { Trampas } \\
\text { noche }\end{array}$} & \multicolumn{3}{|c|}{ Densidad relativa } \\
\hline & & & & & & & Mmus & Rrat & Rnor \\
\hline Alhué Sitio 2 & $\mathrm{RM}$ & $-33,95$ & $-71,03$ & 568 & Ago-03 & 360 & & & 0,3 \\
\hline Coya & VI & $-34,12$ & $-70,32$ & 825 & Nov-02 & 150 & & & 2,7 \\
\hline Reserva Nacional Los Ruiles & VII & $-35,83$ & $-72,51$ & 224 & Jun-03 & 360 & & & 1,4 \\
\hline Parque Nacional Nahuelbuta & IX & $-37,51$ & $-72,55$ & 1.170 & May-03 & 15 & & & 20,0 \\
\hline Parque Nacional Villarrica Sitio 1 & IX & $-39,27$ & $-71,49$ & 1.105 & Jun-00 & 640 & & & 0,2 \\
\hline Parque Nacional Villarrica Sitio 3 & IX & $-39,27$ & $-71,48$ & 1.129 & Abr-01 & 640 & & 0,5 & \\
\hline Parque Nacional Villarrica Sitio 3 & IX & $-39,27$ & $-71,48$ & 1.129 & Nov-01 & 640 & & 0,2 & \\
\hline Parque Nacional Villarrica Sitio 1 & IX & $-39,27$ & $-71,49$ & 1.105 & Feb-02 & 640 & & 0,3 & \\
\hline Parque Nacional Villarrica Sitio 2 & IX & $-39,27$ & $-71,49$ & 1.082 & Feb-02 & 640 & & 0,8 & 0,3 \\
\hline Parque Nacional Villarrica Sitio 3 & IX & $-39,27$ & $-71,48$ & 1.129 & Feb-02 & 640 & & 0,6 & 0,2 \\
\hline Parque Nacional Villarrica Sitio 1 & IX & $-39,27$ & $-71,49$ & 1.105 & Mar-02 & 640 & & 0,3 & \\
\hline Parque Nacional Villarrica Sitio 2 & IX & $-39,27$ & $-71,49$ & 1.082 & Mar-02 & 640 & & 0,3 & \\
\hline Parque Nacional Villarrica Sitio 3 & IX & $-39,27$ & $-71,48$ & 1.129 & Mar-02 & 640 & & 0,6 & \\
\hline Quetrupillan Sitio 2 & IX & $-39,44$ & $-71,79$ & 680 & May-00 & 60 & & & 1,7 \\
\hline Fundo La Montaña & $\mathrm{X}$ & $-39,80$ & $-72,32$ & 125 & Sep-01 & 135 & & 0,7 & \\
\hline Pucatrihue Sitio 1 & $\mathrm{X}$ & $-40,54$ & $-73,71$ & 35 & Oct-03 & 120 & & 0,8 & \\
\hline Pucatrihue Sitio 2 & $\mathrm{X}$ & $-40,54$ & $-73,71$ & 35 & Oct-03 & 360 & & 0,6 & 0,3 \\
\hline Pucatrihue Sitio 3 & $\mathrm{X}$ & $-40,54$ & $-73,71$ & 35 & Oct- 03 & 160 & & & 0,6 \\
\hline Chiloé & $\mathrm{X}$ & $-41,88$ & $-73,67$ & 40 & Ago-00 & 594 & & & 0,2 \\
\hline Chiloé & $\mathrm{X}$ & $-41,88$ & $-73,67$ & 40 & Sep-02 & 720 & & 0,1 & \\
\hline Total roedores recolectados & & & & & & & 24 & 57 & 80 \\
\hline
\end{tabular}

De acuerdo al análisis de las localidades muestreadas, se puede inferir que los roedores cosmopolitas ocupan preferentemente un amplio rango en el gradiente latitudinal de la región mediterránea de Chile. Dentro de la región bioclimática de tendencia tropical (altiplano y prepuna) solo en una localidad (Lasana) se capturó $M$. musculus, mientras que en la región con tendencia oceánica, solo se capturaron roedores murinos en aquella porción con influencia mediterránea (Tabla 2).

\section{Distribución temporal}

La distribución temporal de murinos en Chile central (San Carlos de Apoquindo) se observó estable para los dos representantes de Rattus (Tabla 1). En el caso de M. musculus se observaron episodios de presencia entre enero y mayo de 2000, lo cual coincide con su aparición en otras localidades de Chile central (Tabla 1). Destaca que en ningún caso $M$. musculus aparece junto a representantes de Rattus. Los episodios de aparición de M. musculus fueron seguidos por apariciones de solo $R$. rattus (enero a marzo de 2001), las que luego fueron paulatinamente reemplazadas por $R$. norvegicus. De acuerdo a los datos analizados, $R$. norvegicus apareció en nueve de 32 trampeos en los sitios 1 y 2 , versus $R$. rattus que apareció en seis de 32 trampeos en ambos sitios.

Para el sur de Chile (Parque Nacional Villarrica), la distribución temporal de murinos fue inestable. En efecto, no se registró M. musculus y los dos Rattus ocurrieron fuertemente descontinuados en el tiempo. En la serie temporal se pudo observar un incremento de las poblaciones de $R$. rattus (y secundariamente $R$. norvegicus) a partir de noviembre de 2001 hasta marzo de 2002 (Tabla 1). Luego de este período los muestreos fueron suspendidos por intensas nevadas hasta noviembre de 2002, mes este último en el cual no se capturó ninguna especie de roedor en el Parque. Hasta mayo de 2004 no se han vuelto a recolectar murinos. En el último sitio de muestreo permanente, el Monumento Natural Dos Lagunas en Coyhaique, no se recolectó ningún roedor murino en los 4 años del estudio. 
TABLA 2

Localidades muestreadas para presencia de murinos en Chile. Distancia a centro poblado (DIST) en km. Biótopo se define de acuerdo a Di Castri (1968). Abreviaciones de especies: Mus musculus (Mmus), Rattus rattus (Rrat) y R. norvegicus (Rnor); 0 ausencia, $\mathrm{X}$ presencia

Localities sampled for the occurrence of murine rodents in Chile. Distance to the nearest town (DIST) in kms. Biotope is defined according to Di Castri (1968). Species abbreviations are: Mus musculus (Mmus), Rattus rattus (Rrat) and $R$. norvegicus (Rnor); 0 absence, $\mathrm{X}$ presence

\begin{tabular}{|c|c|c|c|c|c|c|c|c|c|}
\hline Localidad & Región & LAT & LONG & ALT & DIST & Biótopo & & Rrat & Rnor \\
\hline Parinacota & I & $-18,03$ & $-69,27$ & 4.441 & 1 & Tropical de altura & 0 & 0 & 0 \\
\hline Chungará & I & $-18,25$ & $-69,18$ & 4.650 & 10 & Tropical de altura & 0 & 0 & 0 \\
\hline Socoroma & I & $-18,28$ & $-69,59$ & 3.324 & 3 & Tropical de altura & 0 & 0 & 0 \\
\hline Tambo Zapahuira & I & $-18,36$ & $-69,62$ & 3.235 & 15 & Tropical de altura & 0 & 0 & 0 \\
\hline Suricayo & I & $-19,20$ & $-68,89$ & 4.123 & 20 & Tropical de altura & 0 & 0 & 0 \\
\hline Enquelga & I & $-19,22$ & $-68,75$ & 3.850 & 3 & Tropical de altura & 0 & 0 & 0 \\
\hline Lasana, río Loa & II & $-22,16$ & 68,37 & 2.650 & 2 & Tropical marginal & $\mathrm{X}$ & 0 & 0 \\
\hline Parque Nacional Pan de Azúcar & III & $-26,22$ & $-70,59$ & 20 & 17 & Mediterránea perárida & 0 & 0 & 0 \\
\hline Parque Nacional Llanos de Challe & III & $-28,08$ & $-71,14$ & 30 & 3 & Mediterránea perárida & 0 & 0 & 0 \\
\hline Valle del río Tránsito & III & $-28,53$ & $-70,17$ & 1.300 & 5 & Mediterránea perárida & 0 & 0 & $\mathrm{X}$ \\
\hline Observatorio La Silla & IV & $-29,23$ & $-70,74$ & 1.721 & 50 & Mediterránea perárida & 0 & 0 & 0 \\
\hline Parque Nacional Fray Jorge & IV & $-30,67$ & $-71,64$ & 265 & 20 & Mediterránea árida & 0 & 0 & $\mathrm{X}$ \\
\hline Pelambres & IV & $-31,82$ & $-70,58$ & 1.551 & 40 & Mediterránea árida & 0 & 0 & 0 \\
\hline Ñague, Los Vilos & IV & $-31,84$ & $-71,52$ & 78 & 7 & Mediterránea árida & 0 & $X$ & $\mathrm{X}$ \\
\hline Chillepin & IV & $-31,89$ & $-70,80$ & 821 & 21 & Mediterránea árida & 0 & 0 & 0 \\
\hline Cerro Santa Inés, Los Vilos & IV & $-32,16$ & $-71,48$ & 590 & 4 & Mediterránea árida & 0 & 0 & 0 \\
\hline Quebrada del Tigre & $\mathrm{V}$ & $-32,56$ & $-71,44$ & 280 & 1,5 & Mediterránea semiárida & 0 & 0 & 0 \\
\hline Quebrada de Córdoba & $\mathrm{V}$ & $-33,44$ & $-71,65$ & 10 & 2,5 & Mediterránea semiárida & 0 & $X$ & $\mathrm{X}$ \\
\hline Huechún & $\mathrm{RM}$ & $-33,02$ & $-70,82$ & 670 & 4,5 & Mediterránea semiárida & $X$ & 0 & 0 \\
\hline San Carlos se Apoquindo Sitio 1 & $\mathrm{RM}$ & $-33,24$ & $-70,29$ & 1.248 & 2 & Mediterránea semiárida & 0 & 0 & 0 \\
\hline San Carlos de Apoquindo Sitio 2 & $\mathrm{RM}$ & $-33,28$ & $-70,29$ & 1.297 & 2 & Mediterránea semiárida & $\mathrm{X}$ & $X$ & $\mathrm{X}$ \\
\hline El Canelo & $\mathrm{RM}$ & $-33,33$ & $-70,24$ & 1.190 & 3 & Mediterránea semiárida & 0 & $X$ & 0 \\
\hline El Ingenio & $\mathrm{RM}$ & $-33,47$ & $-70,14$ & 1.296 & 2 & Mediterránea semiárida & 0 & 0 & $\mathrm{X}$ \\
\hline Rinconada de Maipú & $\mathrm{RM}$ & $-33,50$ & $-70,91$ & 670 & 10 & Mediterránea semiárida & $X$ & $\mathrm{X}$ & $\mathrm{X}$ \\
\hline El Toyo & $\mathrm{RM}$ & $-33,60$ & $-70,38$ & 928 & 2,5 & Mediterránea semiárida & 0 & 0 & $\mathrm{X}$ \\
\hline Alhué & $\mathrm{RM}$ & $-33,95$ & $-71,03$ & 568 & 12 & Mediterránea subhúmeda & 0 & $\mathrm{X}$ & $\mathrm{X}$ \\
\hline Coya & VI & $-34,12$ & $-70,32$ & 825 & 1 & Mediterránea subhúmeda & 0 & 0 & $\mathrm{X}$ \\
\hline Reserva Nacional Los Ruiles & VII & $-35,83$ & $-72,51$ & 224 & 5,5 & Mediterránea húmeda & 0 & 0 & $\mathrm{X}$ \\
\hline Bullileo & VII & $-36,29$ & $-71,42$ & 650 & 1 & Mediterránea húmeda & 0 & 0 & 0 \\
\hline Parque Nacional Nahuelbuta & IX & $-37,51$ & $-72,55$ & 1.170 & 16 & Mediterránea perhúmeda & 0 & 0 & $\mathrm{X}$ \\
\hline Parque Nacional Huerquehue & IX & $-39,17$ & $-71,73$ & 815 & 5,5 & Mediterránea perhúmeda & 0 & 0 & 0 \\
\hline Parque Nacional Villarrica Sitio 1 & IX & $-39,27$ & $-71,49$ & 1.105 & 14 & Mediterránea perhúmeda & 0 & 0 & 0 \\
\hline Parque Nacional Villarrica Sitio 2 & IX & $-39,27$ & $-71,49$ & 1.082 & 14 & Mediterránea perhúmeda & 0 & 0 & 0 \\
\hline Parque Nacional Villarrica Sitio 3 & IX & $-39,27$ & $-71,48$ & 1.129 & 14 & Mediterránea perhúmeda & 0 & $\mathrm{X}$ & $\mathrm{X}$ \\
\hline Quetrupillan & IX & $-39,44$ & $-71,79$ & 680 & 14 & Mediterránea perhúmeda & 0 & 0 & $\mathrm{X}$ \\
\hline Puesco, Curarrehue & IX & $-39,56$ & $-71,56$ & 1.600 & 25 & Mediterránea perhúmeda & 0 & 0 & 0 \\
\hline Fundo La Montaña & $X$ & $-39,80$ & $-72,32$ & 125 & 8 & $\begin{array}{l}\text { Oceánica con influencia } \\
\text { mediterránea }\end{array}$ & 0 & $X$ & 0 \\
\hline Pucatrihue & $X$ & $-40,54$ & $-73,71$ & 35 & 5 & $\begin{array}{l}\text { Oceánica con influencia } \\
\text { mediterránea }\end{array}$ & 0 & $X$ & $\mathrm{X}$ \\
\hline \multicolumn{10}{|l|}{ La Picada, Parque Nacional } \\
\hline Vicente Pérez Rosales & $\mathrm{X}$ & $-41,03$ & $-72,53$ & 370 & 6 & $\begin{array}{l}\text { Oceánica con influencia } \\
\text { mediterránea }\end{array}$ & 0 & 0 & 0 \\
\hline
\end{tabular}


TABLA 2 (continuación)

\begin{tabular}{|c|c|c|c|c|c|c|c|c|c|}
\hline Localidad & Región & LAT & LONG & ALT & DIST & Biótopo & & Rrat & Rnor \\
\hline Chiloé & $\mathrm{X}$ & $-41,88$ & $-73,67$ & 40 & 5 & $\begin{array}{l}\text { Oceánica con influencia } \\
\text { mediterránea }\end{array}$ & 0 & $X$ & $\mathrm{X}$ \\
\hline Parque Nacional Queulat & $\mathrm{XI}$ & $-44,47$ & $-72,54$ & 114 & 14 & Oceánica templada fría & 0 & 0 & 0 \\
\hline $\begin{array}{l}\text { Reserva Nacional Río Simpson } \\
\text { Monumento Natural }\end{array}$ & $\mathrm{XI}$ & $-45,46$ & $-72,32$ & 210 & 22 & Oceánica templada fría & 0 & 0 & 0 \\
\hline $\begin{array}{l}\text { Dos Lagunas, Sitio } 1 \\
\text { Monumento Natural }\end{array}$ & XI & $-45,31$ & $-71,50$ & 728 & 35 & Oceánica trasandina & 0 & 0 & 0 \\
\hline $\begin{array}{l}\text { Dos Lagunas, Sitio } 2 \\
\text { Monumento Natural }\end{array}$ & XI & $-45,31$ & $-71,50$ & 695 & 35 & Oceánica trasandina & 0 & 0 & 0 \\
\hline Dos Lagunas Sitio 3 & $\mathrm{XI}$ & $-45,32$ & $-71,50$ & 618 & 35 & Oceánica trasandina & 0 & 0 & 0 \\
\hline Mininco & $\mathrm{XI}$ & $-45,51$ & $-71,79$ & 510 & 20 & Oceánica trasandina & 0 & 0 & 0 \\
\hline Fundo El Cóndor, Coyhaique & XI & $-45,56$ & $-71,93$ & 454 & 10 & Oceánica trasandina & 0 & 0 & 0 \\
\hline Lago Atravesado, Coyhaique & $\mathrm{XI}$ & $-45,68$ & $-72,32$ & 339 & 23 & Oceánica subantártica & 0 & 0 & 0 \\
\hline Lago Elizalde & $\mathrm{XI}$ & $-45,79$ & $-72,20$ & 340 & 30 & Oceánica subantártica & 0 & 0 & 0 \\
\hline Fundo El Montoso, Balmaceda & $\mathrm{XI}$ & $-45,96$ & $-71,75$ & 688 & 6 & Oceánica trasandina & 0 & 0 & 0 \\
\hline Alto Río Ibáñez & XI & $-46,09$ & $-72,58$ & 690 & 3 & Oceánica trasandina & 0 & 0 & 0 \\
\hline Lago Cofré, Río Ibáñez & $\mathrm{XI}$ & $-46,19$ & $-72,78$ & 520 & 10 & Oceánica subantártica & 0 & 0 & 0 \\
\hline Puerto Guadal, Chile Chico & XI & $-46,78$ & $-72,60$ & 259 & 3 & Oceánica subantártica & 0 & 0 & 0 \\
\hline Parque Nacional Torres del Paine & XII & $-51,18$ & $-73,04$ & 85 & 50 & Oceánica subantártica & 0 & 0 & 0 \\
\hline
\end{tabular}

\section{Seropositividad}

La búsqueda de anticuerpos mediante Elisa y detección de virus por PCR arrojó resultados positivos en las dos especies de Rattus. De los $57 R$. rattus analizados, un solo ejemplar de la localidad de Ñague, Los Vilos, resultó positivo para la variedad Andes de Hantavirus, y cuyo principal reservorio en Chile es Oligoryzomys longicaudatus (Torres-Pérez et al. 2004, Ortiz et al. 2004). Asimismo, de los 80 R. norvegicus analizados para Andes, dos especímenes resultaron positivos correspondientes a las localidades de Pucatrihue y San Carlos de Apoquindo (M. Ferres resultados no publicados).

Todos los especímenes positivos a virus Hanta, en sus dos variedades, fueron recolectados en ambientes naturales de Chile. Nague (Los Vilos) corresponde a un bosque higrófilo relicto situado en la costa del norte de Chile (Aectoxicum punctatum, Drymis winteri, Luma chequen), y está ubicado a $7 \mathrm{~km}$ de la ciudad de Los Vilos. Pucatrihue se ubica en la costa de la $\mathrm{X}$ Región, y corresponde a un caserío de pescadores cuya vegetación dominante son bosques templados húmedos con especies tales como Drymis winteri; el muestreo se realizó a $5 \mathrm{~km}$ del caserío de pescadores. La tercera forma positiva se registró en San Carlos de Apoquindo.

\section{DISCUSIÓN}

Distribución de murinos en áreas naturales de Chile

La historia natural de los murinos cosmopolitas se asemeja en muchos aspectos a la historia del hombre. Estos roedores han logrado establecerse prácticamente en todo el orbe, causan perturbaciones en los ecosistemas que invaden, trasladan y transmiten enfermedades, establecen relaciones mutualistas con algunas especies, son agresivos con otras, se reproducen todo el año, y hasta han permitido trazar la historia de los desplazamientos humanos en Oceanía (Smith \& Robins 2004). Por ello, llama la atención la poca información disponible sobre su ecología, en especial su rol como especies invasoras, con la salvedad de lo reportado en islas oceánicas donde $R$. rattus, $R$. norvegicus y una tercera forma $R$. exulans han invadido el $82 \%$ de las mayores islas del mundo (Amori \& Clout 2002).

En Chile, M. musculus ocasionalmente se encuentra en ambientes naturales. En las pocas ocasiones que fue recolectado, nunca estuvo asociado a alguna de las especies de Rattus, siendo muy probable que estas últimas lo desplacen. De los tres murinos, solo M. musculus 
fue recolectado en un ambiente de altura, asociado al río Loa (Lasana, II Región, 2.650 m). Lo anterior se confirma con el hallazgo de $M$. musculus a $3.782 \mathrm{~m}$ de altitud en la localidad de Arroyo Coya, también próxima al río Loa (Marquet et al. 1998). Estos últimos autores señalan además otro sitio próximo a Lasana (Chiu-Chiu, $2.500 \mathrm{~m}$ de altitud), donde describen la presencia de $R$. norvegicus pero asociado a presencia humana. Rau et al. (1998) señalaron como dominante la presencia de $M$. musculus y ocasional la de $R$. rattus en la localidad de Opache (2.260 $\mathrm{m}$ de altitud) próxima a la ciudad de Calama. Es probable que en ausencia de Rattus (en ambientes naturales de altura) y frente a crecimientos poblacionales, $M$. musculus tienda a invadir espacios naturales fuera de las viviendas humanas. De hecho, el lugar de captura en Lasana se ubicó a unos $2 \mathrm{~km}$ del pueblo. Otro episodio especial fue lo ocurrido en el veranootoño del 2000 en Chile central, donde M. musculus apareció en tres sitios (Tabla 1). Nuevamente, en ninguna de las localidades se encontró asociado a alguno de los Rattus. El único evento común para estos sitios fue la intensa sequía que afectó a Chile central en ese período.

Distinta es la situación de las dos especies de Rattus, que tienen una extensa distribución en las áreas naturales de Chile. Ambas especies han ocupado exitosamente la región mediterránea y la oceánica con tendencia mediterránea (Di Castri 1968). De acuerdo con las colectas, las barreras naturales para estos invasores son las regiones desérticas del norte, los ambientes de altura y las regiones australes frías. En la zona desértica los Rattus se encuentran asociados a poblaciones humanas (Pine et al. 1979); en localidades del Altiplano no fueron recolectados ni siquiera en lugares próximos a aldeas (Tabla 2), mientras que en las zonas australes existen solo reportes anecdóticos de su presencia en zonas naturales (Figueroa et al. 2001). Otro elemento que influye en la presencia de Rattus en áreas naturales es la distancia a asentamientos humanos. De las tres especies, $R$. norvegicus es el que más se aleja de las construcciones humanas (hasta $20 \mathrm{~km}$ en este estudio). Un hecho destacable es la no tolerancia entre ambos Rattus. Por lo general, ambas especies se pueden registrar juntas, siempre que estén presentes en bajo número. La alta densidad de una de las especies de Rattus es acompañada por la ausencia del otro. Por ejemplo, en el matorral arborescente del llano del Parque Nacional Fray Jorge (IV Región) se recolectaron 17 $R$. norvegicus (septiembre de 2000). Sin embargo, en los fragmentos de bosque de niebla (en la Cordillera de la Costa), $R$. rattus es registrado constantemente (P. Marquet comunicación personal).

Los análisis temporales en San Carlos de Apoquindo y Parque Nacional Villarrica revelan al menos dos características de la distribución de estos murinos en áreas silvestres. La sucesión de estos roedores comprende primero a $M$. musculus, luego a $R$. rattus y por último a $R$. norvegicus. Esto concuerda con los niveles de agresividad que muestran estas especies entre sí (Nolte et al. 2002). En segundo lugar, $R$. norvegicus aparece como dominante en la región mediterránea (San Carlos de Apoquindo), mientras que en la zona sur (Parque Nacional Villarrica) es $R$. rattus quien domina. Lo anterior se relaciona con que $R$. rattus se encuentra de preferencia en zonas más húmedas (bosque de Fray Jorge, bosque húmedo de Los Vilos, Quebrada de Córdova, Parque Nacional Villarrica), mientras que $R$. norvegicus tiene una mayor plasticidad para invadir sectores más secos (valle del río Tránsito, matorral de Fray Jorge, El Toyo), pero que presentan algún curso de agua. La agresividad invasora de $R$. norvegicus se acrecienta al considerar que de los dos Rattus, $R$. norvegicus es el más asociado a cuerpos de agua, por lo que también se le conoce como "rata de alcantarilla". Respecto de la ausencia de Rattus en áreas naturales de la XI Región (e.g., Monumento Natural Dos Lagunas), es muy probable que la rigurosidad climática del lugar sea un factor disuasivo para que los murinos se establezcan en estos ambientes naturales, privilegiando así la estabilidad de los ambientes antrópicos.

\section{Murinos y virus Hanta}

La presencia de virus Hanta variedad Andes ha sido bien establecida para Chile y Argentina (López et al. 1997, Hart \& Bennett 1999, Pavletic 2000, Torres-Pérez et al. 2004, Ortiz et al. 2004). El reservorio principal de este virus corresponde al roedor sigmodontino Oligoryzomys longicaudatus, aunque otras cuatro especies de la misma subfamilia han sido también identificadas como seropositivas 
aunque en baja prevalencia para Chile (Spotorno et al. 2000, Ortiz et al. 2004, E. Palma et al. datos no publicados). El hallazgo de un ejemplar de $R$. rattus infectado con virus Andes (1,75\% de prevalencia en este estudio) genera algunas preguntas tales como ies capaz el virus de pasar de roedores sigmodontinos a murinos?, ¿o solo se trata de un hecho fortuito? $\mathrm{Al}$ respecto, existen evidencias de otro caso en que el virus fue detectado en un murino en el sur de Chile (R. Murúa comunicación personal). Un segundo aspecto es la capacidad de los roedores murinos para diseminar el virus en el ambiente. De ser afirmativas las primeras dos incógnitas, ello constituiría un riesgo epidemiológico serio debido a los hábitos sinantrópicos de estos roedores. La presencia de Rattus infectados revela su interacción con roedores nativos. En la localidad de Los Vilos (IV Región), además de la captura del $R$. rattus contaminado, se recolectaron tres $O$. longicaudatus positivos. Es probable que una alta carga viral en el ambiente haya contaminado a $R$. rattus, aunque la agresividad de esta especie hace muy posible que pudiera haber atacado o depredado a algún roedor infectado. El hallazgo de un $R$. rattus contaminado con Hanta requiere mayor investigación en especial si se considera que ellos pueden interferir con el ciclo silvestre de la enfermedad y luego trasladarse a un dominio más urbano.

La presencia del virus Hanta Seoul en dos ejemplares de $R$. norvegicus constituye el primer reporte de este virus para Chile (Ferres et al. resultados no publicados). De acuerdo con Ulrich et al. (2002), Hanta Seoul pertenece al grupo de virus del Viejo Mundo, los que se caracterizan por causar cuadros de fiebre hemorrágica y síndrome renal (HFRS). Dentro de los Hanta que producen HFRS, Seoul es considerado de mediana patogenicidad con un $1 \%$ de mortalidad (Hart \& Bennett 1999). Este virus ha sido diseminado ampliamente por $R$. norvegicus en Asia, Europa y los Estados Unidos (Hart \& Bennett 1999). La presencia del virus en Chile puede ser explicada al menos por dos vías. Por una parte, las primeras poblaciones invasoras de $R$. norvegicus pudieron haber transportado esta (y otras) enfermedad, y la han mantenido hasta el presente. Por otro lado, el creciente intercambio comercial a nivel global hace posible que a diario seamos invadidos por nuevos especímenes de
Rattus (en especial si se considera el cada vez más creciente comercio con países asiáticos). La baja seropositividad observada $(1,5 \%$ en este estudio), junto al hecho de que no se han reportado patologías en humanos, sugieren que la enfermedad podría haber sido confundida con otras patologías tales como la Leptospirosis, que ocasionan cuadros renales.

\section{Murinos y riesgo ambiental}

La extensiva presencia de Rattus en áreas naturales de Chile representa un riesgo para los ecosistemas invadidos. No obstante, carecemos de antecedentes sobre el impacto de los invasores sobre las comunidades locales. De acuerdo a nuestros resultados, los murinos han invadido de preferencia la región mediterránea de Chile central (Fig. 1). Ello se ha visto favorecido por el alto grado de deterioro ambiental de la región, donde se concentra la mayor parte de la población humana del país. La transformación de espacios naturales en áreas perturbadas antrópicamente genera ambientes que son favorables a los murinos y su proceso expansivo (Nolte et al. 2002). Por otra parte, la región mediterránea en Chile concentra un alto número de especies endémicas, lo que constituye aproximadamente un $50 \%$ del total nacional (Simonetti 1999). Las barreras geográficas del país le confieren a la biota de Chile un carácter insular que puede acrecentar su fragilidad frente a especies exóticas (Jaksic 1998). En islas oceánicas la extinción de reptiles, aves y roedores endémicos ha sido atribuida al impacto de las ratas introducidas (Amori \& Clout 2002). En la Reserva Nacional Los Ruiles en la costa de Chile central (Tabla 2), $\operatorname{dos} R$. norvegicus fueron capturados en el mismo lugar donde se registró un ejemplar del marsupial amenazado Dromiciops gliroides (G. Lobos et al. resultados no publicados). Los hábitos arborícolas de este marsupial pudieron haber evitado la competencia con $R$. norvegicus, sin embargo, si la especie invasora hubiese sido $R$. rattus (de hábitos arborícolas) el impacto podría haber sido mayor.

La relativa cercanía de asentamientos humanos a áreas naturales permite suponer que la mejor herramienta contra las especies invasoras es la conservación de áreas con un alto grado de naturalidad. El alto valor ambiental de la región mediterránea de Chile central, unido a su fuerte grado de perturbación antrópica, ha lle- 


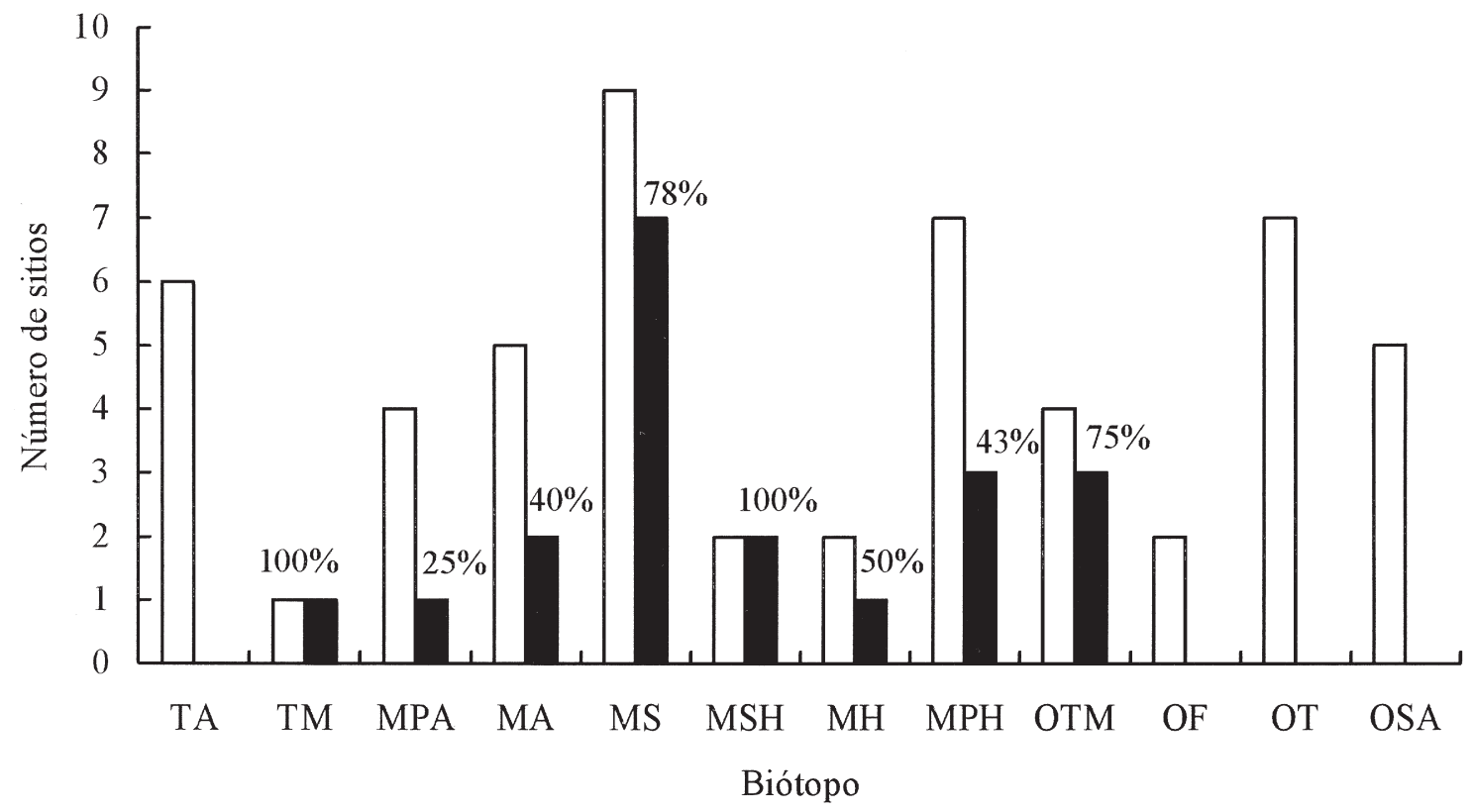

Fig. 1: Ambientes ocupados por roedores cosmopolitas. Las barras blancas indican el total de las localidades prospectadas, las barras oscuras muestran el total de las localidades con presencia de murinos. Abreviaturas corresponden a zonas ecogeográficas: TA tropical de altura, TM tropical marginal, MPA mediterráneo perárido, MA mediterráneo árido, MS mediterráneo semiárido, MSH mediterráneo subhúmedo, MH mediterráneo húmedo, MPH mediterráneo perhúmedo, OTM oceánico con tendencia mediterránea, OF oceánico frío, OT oceánico trasandino y OSA oceánico subantártico (según Di Castri 1968).

Environments occupied by cosmopolitan rodents. The white bars indicate the total of prospected areas, the dark bars show the total of localities with the occurrence of murines. Abreviations are for ecogeographic zones: TA tropical in altitude, TM marginal tropical, MPA mediteranean perarid, MA mediterranean arid, MS mediterranean semiarid, MSH mediterranean subhumid, MH mediterranean humid, MPH mediterranean perhumid, OTM oceanic with mediterranean tendency, OF cold oceanic, OT oceanic transandean, and OSA oceanic subantarctic (sensu Di Castri 1968).

vado a considerarla como uno de los 25 puntos álgidos o "hotspots" para la biodiversidad del planeta (Myers et al. 2000). Por ello, la invasión de roedores murinos en las áreas silvestres de Chile puede constituir una seria amenaza para el patrimonio natural del país.

\section{AGRADECIMIENTOS}

Los autores agradecen a Gabriel Carrasco, Andrés Charrier, Daniel Gómez-Lobo y Fernando Torres-Pérez por el apoyo en el trabajo de campo y a Iván Barría por su colaboración en el manejo de información. También agradecemos los comentarios de dos revisores anónimos que mejoraron versiones previas de este artículo. El presente trabajo fue financiado por el Proyecto "Hantavirus Ecology and Disease in Chile"
(ICIDR Grant NIAID, NIH \# AI 45452), Pontificia Universidad Católica de Chile/The University of New Mexico, USA, y Proyecto FONDECYT 1030488 y FONDECYT-FONDAP 1501-0001 Programa 2.

\section{LITERATURA CITADA}

AMORI G \& M CLOUT (2002) Rodents on island: a conservation challenge. En: Singleton GR, LA Hinds, CJ Krebs \& DM Spratt (eds) Rats, mice and people: rodent, biology and management: 63-68. Australian Centre for International Agricultural Research, Canberra, Australia.

APLIN KP, T CHESSER \& J T HAVE (2002) Evolutionary biology of the genus Rattus: profile of an archetypal rodent pest. En: Singleton GR, LA Hinds, CJ Krebs \& DM Spratt (eds) Rats, mice and people: rodent, biology and management: 487-498. Australian Centre for International Agricultural Research, Canberra, Australia. 
BELL BD (2002) Eradication of alien mammals from five off shores islands Mauritius, Indian Ocean. En: Veitch CR \& MN Clout (eds) Turning the tide: the eradication of invasive species: 40-45. IUCN SSC Invasive Species Specialist Group, International Union for Conservation of Nature, Gland, Switzerland.

CAMPOS H (1996) Mamíferos terrestres de Chile. Guía de reconocimiento. Marisa Cúneo Ediciones, Valdivia, Chile. $221 \mathrm{pp}$.

CAMUS (2005) Introducción de especies en ambientes marinos chilenos: no solo exóticas, no siempre evidente. Revista Chilena de Historia Natural 78: $155-159$

CROSSLAND MR (2000) Direct and indirect effects of the introduced toad Bufo marinus (Anura: Bufonidae) on populations of native anuran larvae in Australia. Ecography 23: 283-290.

DI CASTRI F (1968) Equisse écologique du Chili. Biologie de 1'Amerique australe. En: Debouteville CL \& E Rapaport (eds) Etude sur la faune du Sol. Vol. IV: Biologie de Lamerique Australe: 7-52. Editions du Centre National de la Recherche Scientifique, Paris, France.

FIGUEROA RA, S CORALES, J CERDA \& H SALDIVIA (2001) Roedores, rapaces y carnívoros de Aysén. Servicio Agrícola y Ganadero, Gobierno Regional de Aysén, Coyhaique, Chile. 197 pp.

GARCÍA MA, CE DIEZ \& AO ÁLVAREZ (2002) The eradication of Rattus rattus from Monito Island, West Indies. En: Veitch CR \& MN Clout (eds) Turning the tide: the eradication of invasive species: 116-199. IUCN SSC Invasive Species Specialist Group, International Union for Conservation of Nature, Gland, Switzerland.

HART CA \& M BENNETT (1999) Hantavirus infections: epidemiology and pathogenesis. Microbes and Infection 1: 1229-1237.

JAKSIC F (1998) Vertebrate invaders and their ecological impacts in Chile. Biodiversity and Conservation 7: 1427-1445.

KATS LB \& RP FERRER (2003) Alien predators and amphibian declines: review of two decades of science and the transition to conservation. Diversity and Distribution 9: 99-110.

LÓPEZ N, P PADULA, C ROSSI, S MIGUEL, A EDELSTEIN, E RAMÍREZ \& MT FRANZEFERNÁNDEZ (1997) Genetic characterization and phylogeny of Andes virus and variants from Argentina and Chile. Virus Research 50: 77-84.

MARQUET P, F BOZINOVIC, GA BRADSHAW, C CORNELIUS, H GONZÁLEZ, JR GUTIÉRREZ, ER HAJEK, JA LAGOS, F LÓPEZ-CORTÉS, L NÚÑEZ, EF ROSELLO, C SANTORO, H SAMANIEGO, VG STANDEN, JC TORRES-MURA \& FM JAKSIC (1998) Los ecosistemas del desierto de Atacama y área andina adyacente en el norte de Chile. Revista Chilena de Historia Natural 71: 593-617.

MAZZONI R, AA CUNNINGHAM, P DASZAK, A APOLO, E PERDOMO \& G SPERENZA (2003) Emerging pathogen of wild amphibians in frog (Rana castesbeiana) farmer for internacional trade. Emerging Infectious Diseases 9: 995-998.

MICOL T \& P JOUVENTIN (2002) Eradication of rats and rabbits from Saint-Paul Island, French Southern Territories. En: Veitch CR \& MN Clout (eds) Turning the tide: the eradication of invasive species: 199-205. IUCN SSC Invasive Species Specialist Group, International Union for Conservation of Nature, Gland, Switzerland.
MILLER S \& J ROTTMANN (1976) Guía para el reconocimiento de mamíferos de Chile. Editorial Gabriela Mistral, Santiago, Chile. 200 pp.

MILLS J, J CHILDS, T KSIAZEK \& C PETERS \& W VALLECA (1995) Methods for trapping and sampling small mammals for virologic testing. United States Department of Health and Human Services, Atlanta, Georgia, USA. 61 pp.

MUÑOZ-PEDREROS A \& J YÁÑEZ (eds) (2000) Mamíferos de Chile. CEA ediciones, Valdivia, Chile. 464 pp.

MYERS N, RA MITTERMELER, CG MITTERMELER, GAB DA FONSECA \& J KENTS (2000) Biodiversity hotspots for conservation priorities. Nature 403: 853- 858.

NOLTE D, D BERGMAN \& J TOWNSEND (2002) Roof rat invasion of an urban desert island. En: Singleton GR, LA Hinds, CJ Krebs \& DM Spratt (eds) Rats, mice and people: rodent, biology and management: 481-484. Australian Centre for International Agricultural Research, Canberra, Australia.

NÚÑEZ F \& P CISTERNAS (1991) Roedores domésticos I. Caracterización morfológica conductual y sanitaria. Monografías Medicina Veterinaria 13: 5564.

ORTIZ JC, W VENEGAS, JA SANDOVAL, P CHANDIA \& F TORRES-PÉREZ (2004) Hantavirus en roedores de la Octava Región de Chile. Revista Chilena de Historia Natural 77: 251-256.

PEFAUR JE, F JAKSIC F \& JL YÁÑEZ (1978) Estudio poblacional de Mus musculus silvestre en Santiago, Chile. Publicación Ocasional, Museo Nacional de Historia Natural (Chile) 26: 1-12.

PAVLETIC C (2000) Hantavirus: su distribución geográfica entre los roedores silvestres de Chile. Revista Chilena de Infectología 17: 186-196.

PINE RH, SD MILLER \& ML SCHAMBERGER (1979) Contributions to the Chilean mammalogy. Mammalia 43: 339-376.

RAU JR, C ZULETA, A GANTZ, F SAIZ, A CORTÉS, L YATES, AE SPOTORNO \& E COUVE (1998) Biodiversidad de artrópodos y vertebrados terrestres del Norte Grande de Chile. Revista Chilena de Historia Natural 71: 527-554.

ROBERTSON HA \& EK SAUL (2004) Conservation of kakerori (Pomarea dimidiata) on the Cook Islands 2002/2003. DOC Science Internal Series 167, Department of Conservation, Wellington, Australia. $16 \mathrm{pp}$.

RODRÍGUEZ JA (1993) Roedores plagas: un problema permanente en América Latina y el Caribe. Oficina regional de la FAO para América Latina y el Caribe, Santiago, Chile. 85 pp.

RODRÍGUEZ JP (2001) Exotic species introductions into South America: an underestimated threat? Biodiversity and Conservation 10: 1983-1996.

SCHMALJOHN C \& B HJELLE (1997) Hantaviruses: a global disease problem. Emerging Infectious Diseases 3: 95-104

SIMONETTI JA (1983) Occurrence of the black rat (Rattus rattus) in central Chile. Mammalia 47: 131132.

SIMONETTI JA (1999) Diversity and conservation of terrestrial vertebrates in mediterranean Chile. Revista Chilena de Historia Natural 72: 493-500.

SMITH EM \& JH ROBINS (2004) Origins and dispersals of Pacific people: evidence from mtDNA phylogenies of the Pacific rat. Proceedings of the National Academy of Sciences USA 101: 91679172 . 
SPOTORNO A, RE PALMA \& J VALLADARES (2000)

Biología de roedores reservorios de Hantavirus en Chile. Revista Chilena de Infectología 17: 197210.

SUTHERLAND WJ (2001) The conservation handbook. Research, management and policy. Blackwell Science Ltd., London, United Kingdom. 278 pp.

TORRES-PÉREZ F, J NAVARRETE-DROGUETT, R ALDUNATE, TL YATES, GJ MERTZ, P VIAL, M
FERRES, P MARQUET \& RE PALMA (2004) Peridomestic small mammals associated with confirmed cases of human hantavirus disease in southcentral Chile. American Journal of Tropical Medicine and Hygiene 70: 305-309.

ULRICH R, B HJELLE, C PITRA \& DH KRUGER (2002) Emerging viruses: the case "Hantavirus". Intervirology 45: 318-327.

Editor Asociado: Luis Ebensperger

Recibido el 19 de agosto de 2004; aceptado el 21 de octubre de 2004 